\title{
Soft Rabatics
}

2 Closed-loop haptic feedback control using a self-sensing soft pneumatic actuator skin

3

4

5

6

7

8

9

10

Harshal A. Sonar ${ }^{1}$, Aaron P. Gerratt ${ }^{2}$, Stéphanie P. Lacour ${ }^{2}$, Jamie Paik $^{1, *}$

1 Reconfigurable Robotics Laboratory, Ecole Polytechnique Fédérale de Lausanne, 1015 Lausanne, Switzerland

2 Laboratory for Soft Bioelectronic Interfaces, School of Engineering, Center for Neuroprosthetics, EPFL

*jamie.paik@epfl.ch

Keywords: Wearable technology, tactile feedback device, liquid metal sensor, strain sensor; soft pneumatic actuators, haptic device.

\section{Abstract:}

In this manuscript, we achieve a closed-loop control over haptic feedback, first time for an entirely soft platform. We prototyped a novel self-sensing soft pneumatic actuator (SPA) with soft strain sensors, called SPA-skin, that withstands large multiaxial strains and is capable of high frequency sensing and actuation. To close-loop control the haptic feedback, the platform requires a cohesively integrated system. Our system consists of a stretchable low profile $(<500$ $\mu \mathrm{m})$ SPA and an ultra-compliant thin-metal film strain sensor that create a novel bidirectional platform for tactile sensing via force-tunable vibratory feedback. With this prototype, we demonstrated control of the actuator shape in real-time up to $100 \mathrm{~Hz}$ at output forces up to $1 \mathrm{~N}$, maintained under variable mechanical loadings. We further characterized the SPA-skin platform for its static and dynamic behavior over a range of actuation amplitude and frequencies as well as developed an analytical model of this system to predict the actuator inflation state only using the embedded sensor's resistance. Our SPA-skin is a multifunctional multilayer system that can readily be implemented as a high-speed wearable bidirectional interface for contact sensing and vibrotactile feedback. 


\section{Soft Robotics}

\section{Objective:}

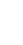

Human haptic perception, in addition to visual and auditory feedback, plays a key role in understanding and exploring our local environment. ${ }^{[1]}$ Desktop-scale haptic devices specifically dedicated for providing tactile stimulation have proven their efficacy in various applications including medical rehabilitation, ${ }^{[2]}$ medical technologies, ${ }^{[3]}$ entertainment, and virtual or augmented reality. ${ }^{[4]}$ More compact vibratory haptic actuators have been integrated into system for human machine interactions such as, car steering wheels, and joysticks; ${ }^{[5],[6]}$ however, these implementations provide limited physical feedback such as in mobile phones and joysticks that can only modulate its frequency. Furthermore, this frequency modulation is not feedback controlled.

For wearable technology to have accurate physical feedback to the wearer, feedback controlled actuation is needed. As such, the requirements for wearable devices, which include adaptability and conformability to the body of a human wearer, extends to the haptic technology itself. Existing haptic technologies, however, do not meet the physical requirements necessary to achieve these characteristics. Conformable interfaces interacting with human skin should be made of thin elastic materials that have moduli similar to skin, $0.3-2 \mathrm{MPa} .^{[7]-[11]}$ This way, the interface can conform to the body and still operate over a wide frequency range $(1-400 \mathrm{~Hz})$ and forces up to $1 \mathrm{~N}^{\left[{ }^{[12]}\right.}$ Available tactile feedback devices primarily rely on rigid piezoelectric elements or electromechanical eccentric mass motors. ${ }^{[4],[6]}$ Compliant actuators can be developed with elastomeric materials such as soft silicones that have elastic moduli similar to human skin. ${ }^{[7]}$, ${ }^{[8],[11]}$ The softness and compliance of the soft robotic systems have been useful in many wearable applications ranging from assistive technologies, medical rehabilitation to entertainment. ${ }^{[13]-[18]}$ Vibro-tactile displays using electro-active polymer (EAP) based soft actuators have tackled many of the challenges associated with conventional, rigid vibro-tactile devices, but provide limited wearability as they require high-voltages. ${ }^{[12],[13]}$ Soft material-based pneumatic actuators, composed of elastically inflatable fluidic chambers, have demonstrated their benefits in robotic applications through large-scale deformation and high compliance, and have potential to be adapted for wearable haptic applications. ${ }^{[21]-[28]}$ The major shortcomings for the pneumatic powered actuators is the size of the power supply and tube-length limiting the bandwidth for high-frequency of actuation which need to be separately addressed. 


\section{Soft Rabatics}

1 Wearable haptic devices need to ensure that the force imparted on the wearer is consistent with respect to the perceived performance: this depends on how the interface fits and moves with the body. The effective tactile feedback also requires accurate and coherent force transfer from the actuator to the wearer regardless of the external loading or manufacturing variations. This requires embedded sensing of the actuator state and corresponding corrective measures through closed-loop control. There has been significant development in entirely soft and stretchable physical sensors to measure crucial parameters like strain and pressure experienced. [13], [16], [29]${ }^{[32]}$ Advanced manufacturing technologies like soft 3D printing have combined soft sensing within soft actuation. ${ }^{[33]}$ Such an integration of soft sensing with soft actuation creates possibility of closed-loop control which is essential to adapt to the external loading conditions suitable for wearable scenarios. However, no wearable haptic device has demonstrated closed-loop control capabilities with embedded sensing. It has been particularly difficult to design soft stretchable sensors, which can perform under high strains and high strain rates needed for state estimation of high-speed vibratory actuation.

We feedback controlled for the first time, an entirely soft haptic platform. We experimented this on a novel platform composed of a soft sensor integrated soft pneumatic actuator (SPA): SPAskin. Its entirely soft design enables flexible, conformal, and distributable interface over a variety of surfaces. In addition, the integrated sensing and active closed-loop control provide accurate modulation of actuation amplitude and frequency for rich feedback, independent of an external loading.

The major contributions of this work include:

1. Active closed-loop control of soft haptic platform independent of the loading conditions. The closed-loop control compensates for the external disturbance when occurred; therefore, the produced haptic feedback is consistent and accurate.

2. Design of a novel haptic platform that is entirely soft, based on a pneumatic actuation and integrated stretchable liquid metal sensors.

3. Characterization of SPA-skin for high-fidelity $(0-100 \mathrm{~Hz})$ operation and analytical model to estimate the actuator inflation state using sensor resistance. 


\section{Soft Robotics}

\section{Materials and Methods} $100 \mathrm{~Hz}$.

\section{SPA-skin design and manufacturing:}

The SPA-skin interface, a sensor and actuator laminate, is specifically designed to provide a consistent vibrotactile feedback under variation of the input parameters: amplitude, vibratory frequency, and external loading force (Figure 1). Amplitude and frequency are the inputs set by the user-need, while external loading is dictated by the environmental interactions and disturbance. The sensor-integrated SPA compensates for these three inputs through an active closed-loop feedback control. Here, a seamlessly integrated stretchable strain sensor measures the strain experienced upon the actuator inflation by the resistance change. In case of external loading, the strain value measured is maintained to the set point by actively controlling the input pressure. We also developed a custom data acquisition platform to enable strain sensing and actuation control at high-speed $(1 \mathrm{kHz})$ to reliably cover the SPA actuation frequency from 0 to

The SPA-skin - a sensor-actuator laminate- is composed of soft silicone elastomers and thin biphasic metal films, as depicted Figure 2a. The actuator layer, SPA, consists of an elastomeric membrane that can be pneumatically inflated with a positive pressure input. ${ }^{[34]}$ This actuator is fabricated with three thin layers: a middle flexible mask layer $(50 \mu \mathrm{m})$ to define the actuator's shape, sandwiched between two silicone layers, with a total thickness of $500 \mu \mathrm{m} .{ }^{[34]}$ The masking layer is laser cut to obtain the desired shape and is then laminated onto the bottom silicone layer to be encapsulated by a thin top silicone layer. The polypropylene mask adheres to the bottom silicone and ensures that, upon inflation, deformation occurs in the top membrane. The actuator geometry is governed by the spatial resolution requirement of $20 \mathrm{~mm}$ and force requirement of

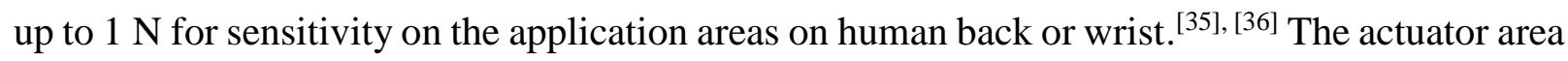
is $10 \mathrm{~mm}$ in diameter and has a $2 \mathrm{~mm}$ inlet tube. The sensor layer, an eGaIn filled micro channel membrane, is prepared with a biphasic (liquid-solid) Gallium-based metallization patterned on a thin silicone membrane. ${ }^{[30]}$ It is then laminated on top of the actuator to complete the soft actuator-sensor interface. The layout of the strain sensor covers the entire area of the SPA in order to record changes in strain upon inflation with positive input pressure. The soft sensor-skin hosts metallic meanders on a $40 \mu \mathrm{m}$ thick (substrate and encapsulation) on Polydimethylsiloxane (PDMS) film patterned at the resolution limit of the biphasic metallization technique in order to maximize the sensor sensitivity. The entire SPA-skin is fabricated using PDMS (Dow Corning Sylgard 184) because of its low viscoelasticity ${ }^{[37]}$ and because multiple layers can be covalently 


\section{Soft Robatics}

1 bonded with oxygen plasma treatment. The intentional use of the same material for both the

actuator and the sensor substrate yields a robust monolithic functional skin with embedded sensing and actuation capabilities. The softness of SPA-skin manufactured matches with that of the human skin to have a compliant and wearable prototype.

\section{SPA-skin characterization}

The frequency range of somatic perception for human skin varies from $0-100 \mathrm{~Hz}$ for rapidly adapting sensor receptors and $100-400 \mathrm{~Hz}$ Pacinian corpuscles. ${ }^{[12]}$ In order to apply a controlled feedback of the integrated SPA-skin system over such a variety of modulable amplitude and frequency ranges, an accurate model of the steady state and transient behavior is first needed. We, therefore, perform static and dynamic characterization of the SPA-skin to obtain all relevant system parameters necessary for implementing closed-loop control.

Static characterization Despite the addition od extra sensor layer, we observed a minimal mechanical loading on the SPA with less than $10 \%$ change in the actuator inflation (Figure $\mathbf{2 b}$ ). The covalent bonding between sensor and actuator layers ensures the conformity of the sensor layer around the SPA during inflation. This enables the accurate measure of the strain experienced by the SPA through the soft sensors, even at high strain rates. Upon inflation of the SPA, the resistance of the soft sensor increases due as the biaxial strain increases. The gauge factor (GF) obsereved for the stretchable metallization is approximately unity and independent of the strain rate [see the supplementary material]. The SPA-skin system exhibited a robust behaviour, with less than $10 \%$ deviation in the relative change in resistance $(\Delta R / R)$ obtained during high strain rate operation at $20 \mathrm{~Hz}$ to a pressure of $25 \mathrm{kPa}$ for a million cycles (Figure 2c). Such a robustness highlights the ability of the SPA-skin to be used in the wearable applications over long duration under dynamic operation. The sensors also demonstrate rapid response times under $1 \mathrm{~ms}$, meeting the demands of the high frequency action for vibrotactile feedback. During the cyclic test of 1 million cycles at $20 \mathrm{~Hz}$, a $10 \%$ change of resistance per cycle was observed. This corresponds to an average strain rate of $400 \% / \mathrm{s}(10 \% \times 2 \times 20 \mathrm{~Hz})$ experienced by the SPA-skin for a million cycles, with continuous current consumption at $1.25 \mathrm{~mA}$ throughout the $14 \mathrm{~h}$ of testing period.

Dynamic characterization Our SPA-skin can modulate the actuation amplitude over $100 \mathrm{~Hz}$ of dynamic range of frequencies derived from human mechano-reception capabilities. The SPAskin is actuated with an on-off pressure input that is regulated at set amplitudes between, $5 \mathrm{kPa}$ and $30 \mathrm{kPa}$. The dynamic response shows an increase in the relative change in resistance, $\Delta R / R_{0}$, concomitant to the increase of the actuation pressure at $5 \mathrm{~Hz}$ (Figure 2d). The inflation 


\section{Soft Robotics}

and deflation behavior of the sensor-actuator system is repeatable over a range of actuation frequencies, as seen in the five consecutive cycles shown in Figure 2c. The system dynamics resulting from the material elastic properties and the actuator geometry with $\phi 2 \mathrm{~mm}$ and tube length of $300 \mathrm{~mm}$, limit the maximum rate of change in inflation/deflation. This manifests as an increase in the baseline and decrease in the maximum output as the operational frequency increases (Figure 2e). The cutoff frequency of our SPA-skin design is measured to be $30 \mathrm{~Hz}$, above which the actuation amplitude is less than $70 \%$ of its maximum value. Although the vibration amplitude decreases above $30 \mathrm{~Hz}$, it is still measurable and perceivable to the human skin for the frequencies up to $100 \mathrm{~Hz}$ (Figure 2f). ${ }^{[35]}$ The measurement system can measure the resistance of sensor dynamically with accuracy of $0.4 \%$ at 1600 samples per second which enabled recording of 16 samples at $100 \mathrm{~Hz}$. This level of accuracy allowed for measurement of 25 distinct levels for $10 \%$ change in SPA strain.

\section{Analytical model and Control strategies}

The static and dynamic characteristics of SPA-skin show the integrated system is repeatable and stable over a range of actuation frequencies and amplitude. The sensor feedback therefore can be used to actively control the SPA inflation height if the relationship between actuation amplitude and measured resistance can be developed. It is also important to note that the soft actuators exhibit complex mechanical behaviour upon inflation due to the hyper-elastic nature of the silicone materials. Such a behaviour makes it complicated to accurately maintain the actuator inflation amplitude or output blocked force only with open-loop analytical model without a feedback sensing mechanism. We utilized the integrated resistive strain sensors to predict the average strain experienced by actuator and map it to the actuation height. We developed and experimentally verified an analytical model for the actuator behaviour upon inflation, based on the results obtained from the integrated resistive sensor-skin, an external inflation height sensor, and an external pressure sensor (Figure 3a). Our model maps the measured resistance change $\left(\Delta R / R_{0}\right)$ from sensor to inflation height $(h)$ analytically, based on the actuator inflation geometry and biaxial deformation of material upon inflation. In the first stage we developed geometrical mapping function for the measured strain for a given inflation height (Figure 3a). As the strain experienced by the sensor meanders is equibiaxial, we then map it to corresponding change in resistance value resulting from the actuator inflation. 


\section{Soft Rabatics}

1 Assuming a Poisson's ratio $(v)$ of 0.5 for PDMS and an isotropic nature of the soft material, we can assume equibiaxial stress in the actuator membrane upon inflation. Also, the effect of reduction in the remaining dimensions $\left(-\varepsilon^{\prime}\right)$ for applied strain $(\varepsilon)$ in one dimension for a given Poisson's ratio is:

$$
\left(1-\varepsilon^{\prime}\right)=(1+\varepsilon)^{-v}
$$

Therefore, the change in dimensions of a single meander channel with length $\left(l_{0}\right)$, width $\left(w_{0}\right)$, and thickness $\left(t_{0}\right)$ from equibiaxial strain upon inflation $\left(\varepsilon=\varepsilon_{l}=\varepsilon_{w}\right)$ can be given as:

$$
\begin{gathered}
l=l_{0}+\Delta l_{l}-\Delta l_{w}=l_{0}\left(1+\varepsilon_{l}-{\varepsilon^{\prime}}_{w}\right)=l_{0}\left[(1+\varepsilon)^{-v}+\varepsilon\right] ; \\
w=w_{0}-\Delta w_{l}+\Delta w_{w}=w_{0}\left(1-\varepsilon^{\prime}{ }_{l}+\varepsilon_{w}\right)=w_{0}\left[(1+\varepsilon)^{-v}+\varepsilon\right] ; \\
t=t_{0}-\Delta t_{l}-\Delta t_{w}=t_{0}\left(1-\varepsilon^{\prime}{ }_{l}+1-\varepsilon^{\prime}{ }_{w}-1\right)=t_{0}\left[2(1+\varepsilon)^{-v}-1\right]
\end{gathered}
$$

Where, $\Delta l_{l / w}, \Delta w_{l / w}$ and $\Delta t_{l / w}$ are changes in length, width and height of the soft material due to strain in the direction of length/width $(l / w)$, respectively.

Hence, the change in resistance of the biphasic metal encapsulated within the silicone membranes can be given as:

$$
\frac{R}{R_{0}}=\frac{l / l_{0}}{\left(w / w_{0}\right)\left(t / t_{0}\right)}=\frac{1}{2(1+\varepsilon)^{-v}-1}
$$

Furthermore, the correlation between uniaxial strain and actuator inflation height can be formulated using the volumetric change in the actuator and spatial distribution of sensor meanders on the actuator. The actuator of diameter $(d)$ inflated to height $(h)$ takes the approximate shape of a scooped sphere with a variable radius ( $r$ ) (Figure 3a, b). The radius decreases from infinity to $5 \mathrm{~mm}$ as actuator inflates from $0 \mathrm{~mm}$ to $5 \mathrm{~mm}$ height. The average uniaxial strain in a single meander during inflation to height can be described as:

$$
\varepsilon=\frac{l-l_{0}}{l_{0}}=\frac{\sin ^{-1} \frac{x}{r}}{\frac{x}{r}}-1
$$

Where, $x$ is the actuator radius, $l$ is the final length, $l_{0}$ is initial length, and $\mathrm{r}=\sqrt{\mathrm{x}^{2}+(\mathrm{r}-\mathrm{h})^{2}}$. The sensor meander pattern consists of 18 parallel lines equally distribute along the surface of the actuator membrane. The $i^{t h}$ stripe of the sensor channel is distance $\left(d_{i}\right)$ from center of the actuator and during inflation reaches height $\left(h_{i}\right)$ :

$$
h_{i}=h_{\max }-r+\sqrt{r^{2}-d_{i}^{2}}
$$

From Equations 3 and 4, the average strain experienced by all the sensor meanders on the actuator surface is given as: 


\section{Soft Robotics}

$$
\varepsilon_{\mathrm{avg}}=\sum_{\mathrm{i}=1}^{\mathrm{n}} \frac{\varepsilon_{\mathrm{i}}}{\mathrm{n}}=\frac{1}{\mathrm{n}} \sum_{\mathrm{i}=1}^{\mathrm{n}}\left\{\frac{\sin ^{-1} \frac{x}{r_{i}}}{\frac{x}{r_{i}}}-1\right\} \approx \frac{\Delta R}{R}
$$

2 Where, the GF is $1 .^{[30]}$

Hence, to deduce the inflation height $(h)$ by measuring change in resistance $\left(\Delta R / R_{0}\right)$ an explicit mapping from $\Delta \mathrm{R} / R_{0} \rightarrow h$ is necessary. However, it is impossible to obtain, as Equation 2 is a coupled equation. Therefore, a piecewise polynomial curve fit is obtained for the inverse mapping of Equation 2 (Figure 3c):

$$
\begin{gathered}
h=49800\left(\frac{\Delta R}{R_{0}}\right)^{3}-2890\left(\frac{\Delta R}{R_{0}}\right)^{2}+74.6\left(\frac{\Delta R}{R_{0}}\right)+0.109 \text { for } 0<\left(\frac{\Delta R}{R_{0}}\right)<0.025 \\
h=33\left(\frac{\Delta R}{R_{0}}\right)^{3}-31\left(\frac{\Delta R}{R_{0}}\right)^{2}+16\left(\frac{\Delta R}{R_{0}}\right)+0.63 \text { for } 0.025<\left(\frac{\Delta R}{R_{0}}\right)<0.4
\end{gathered}
$$

with Error Norm $=0.0079$.

\section{Results}

Experimental setup

High-speed actuation of SPA-skin demands a test setup that provides a high data rate resistance measurement and a low settling time pressure regulation. We required at least 1000 samples/second of sensor resistance values in order to ensure reliable and accurate estimation of actuator state, as a digital control system needs at least 8-10 times more samples than the actual operational frequency of the system (100 Hz for SPA-skin). We developed a custom 16-bit highspeed data acquisition system with a low on-resistance multiplexer, A/DC (ADS8517, TI, USA) and an Arduino microcontroller board that measured the sensor resistance at $1600 \mathrm{~Hz}$ per channel with $0.4 \%$ accuracy (Figure 3a). The low resistance multiplexer reduced the effect of extra resistance added in the sensor resistance measurement. It was also necessary to have a pressure regulator that can react within $10 \mathrm{~ms}$ to be able to control the SPA-skin at $100 \mathrm{~Hz}$ of vibratory output. In practice, we obtained settling time of $20 \mathrm{~ms}$ with a piezo-actuator drive pressure for maximum pressure change from 0 to $100 \mathrm{kPa}$ that allowed us to control SPA-skin up to $50 \mathrm{~Hz}$. The Arduino microcontroller also directed the control commands to this piezo-pressure regulator to achieve the desired set point. The test setup is also equipped with an integrated pressure sensor (001BGAA5, Honeywell) and a laser interferometer (HG-C1030, Panasonic) to measure the SPA pressure and the inflation height, respectively. There is a micro-meter placed vertically and connected to a high precision 3D force sensor (Nano 17, ATI industrial automation, USA) for 


\section{Soft Robotics}

applying a controlled blocked force externally to simulate disturbances.[supplementary Figure S3c].

\section{Model validation}

Our test setup enabled measurement of inflation height, input pressure and strain sensor resistance to process the data for validation of the analytical model developed in previous section. We measured the maximum height $\left(h_{\max }\right)$ during inflation at the given pressure with an external laser displacement sensor and recording the sensor response as depicted in Figure 3b. We inflated SPA-skin at various input pressure and took images for fitting a curve of radius ' $r$ ' and while simultaneously measuring the observed inflation height $(h)$. The experimental values of curved fitted radii with the scooped sphere approximation for given inflation height matches closely with the expected geometrical model (Figure 3b). Afterwards, we derived the average strain values from the Equation 5 and the external height sensor reading. Assuming the GF of 1 we obtained the analytical model plot of expected resistance change $\left(\frac{\Delta R}{R}\right)$ for given inflation height in Figure 3c. The subsequent experimental measurements shows the resistance change over inflation height of SPA-skin for three different samples are well in agreement with the analytical model (Figure 3c) [and supplementary Figure S2]. This confirmed the integrated strain sensor could effectively be used to measure the actuator inflation height, eliminating the dependency of actuator performance on the manufacturing variations and input pressure. Next, we developed a strain controller to have the strain sensor for active closed loop control.

\section{Active control of the force exerted by the SPA-skin}

A coherent and responsive haptic feedback from deformable soft interfaces relies on explicit control of the actuation force and frequency, regardless of the loading conditions. If not, the wearer will feel unstable feedback depending on how the device is positioned. Hence, we demonstrated the closed-loop capabilities to actively adapt for the changes in the loading and provide a coherent tactile feedback. We designed a setup which uses a $1 \mathrm{~cm}^{3}$ cube of Ecoflex 30 silicone ${ }^{[8]}$ to simulate contact with the human skin, placed over the SPA-skin (Figure 4a). Ecoflex 30 is selected as its compressive behavior is comparable with that of human skin. ${ }^{[38]}$

We have the relationship between the measured resistance and the biaxial strain on the system from the analytical model. The biaxial strain maintained under uniform soft loading will provide compensation for the added pressure through increase in the input pressure. 


\section{Soft Robotics}

1 A PID controller is developed with desired resistance as setpoint to control the input pressure and eventually the inflation amplitude to demonstrate the closed-loop control capabilities of the SPA-skin. During free, unloaded operation, the control loop easily achieved the arbitrary strain set points (Figure 4b). Additionally when external forces were introduced, the system continues to converge to the set point inflation, regardless of the pre-load (Figure $4 \mathbf{c}$ ). The feedback from the embedded strain sensors guarantees the desired actuation, even when the external loading is an order of magnitude higher than the desired blocked-force produced by the SPA-skin (Figure 4d). Eventually, as the loading increases, the system's mechanical inertia increases, which leads to an increase in the overshoot in the control (Figure 4c). We tuned the PID gains conservatively to cover this fact and compensate for the overshoot, while degrading a little on the system settling time.

\section{Conclusion}

For an effective wearable technology, we require an accurate understanding of the physical interactions between the device and the wearer's perception. We engineered a closed-loop control of an entirely soft wearable haptic platform over a wide range of vibrotactile feedback. The soft material based device design provided inherent safety much needed for human interaction as well as compliance matching with human skin to provide natural wearability.

We prototyped a soft monolithic sensor integrated SPA with embedded sensing and actuation capabilities, and with soft sensors that have no mechanical loading impact on the actuator. Use of SPAs provided high actuation force, wide range of actuation frequency, simple design for iteration and a highly customizable thin platform as opposed to the traditionally available rigid vibration motors. The SPA-skin produced forces up to $1 \mathrm{~N}$ with controllability of $0.05 \mathrm{~N}$, and actuation dynamics of up to $100 \mathrm{~Hz}$ with controllability of $0.1 \mathrm{~Hz}$; these are essential for providing a wide range of proprioceptive feedback to the wearer. The system characterization of SPA-skin under continuous actuation and sensing showed robust and repeatable behavior over 1 million cycles thereby promising compatibility with "real life" wearable applications. We further demonstrated closed-loop control capabilities of this SPA-skin system at $15 \mathrm{~Hz}$ sinusoidal vibration at $1 \mathrm{kHz}$ control speed. The closed-loop controller, based on an analytical model of the system, uses the sensor resistance to control the average strain of the actuator and allow for generating uniform output force under different loading conditions. This allowed the wearer to feel a consistent feedback independent of the wearing conditions. Furthermore, the bidirectional 


\section{Soft Robotics}

operations of the SPA-skin platform offer a wearable system that can not only be used for haptic feedback but also for quantification of human proprioceptive capabilities over range of frequencies. This makes SPA-skin a promising solution for applications demanding more intuitive and comprehensive tactile interactions. Specifically SPA skin allows user studies can benefit from this quantitative measurement of feedback through integrated soft sensing to match with user's qualitative responses over tactile feedback.

$<<$ talk about SPA-skin Array $>>$

\section{Acknowledgement}

The authors would like to thank the Swiss National Centre of Competence in Research (NCCR) in Robotics, the European Research Project Proof of Concept grant (SoWe), and the Bertarelli Foundation.

\section{References:}

[1] J. Minogue and M. G. Jones, "Haptics in Education: Exploring an Untapped Sensory Modality," Rev. Educ. Res., vol. 76, no. 3, pp. 317-348, Jan. 2006.

[2] R. Sigrist, G. Rauter, R. Riener, and P. Wolf, "Augmented visual, auditory, haptic, and multimodal feedback in motor learning: A review," Psychon. Bull. Rev., vol. 20, no. 1, pp. 21-53, Feb. 2013.

[3] E. P. Westebring-van der Putten, R. H. M. Goossens, J. J. Jakimowicz, and J. Dankelman, "Haptics in minimally invasive surgery--a review," Minim. Invasive Ther. Allied Technol. MITAT Off. J. Soc. Minim. Invasive Ther., vol. 17, no. 1, pp. 3-16, 2008.

[4] A. U. Alahakone and S. M. N. A. Senanayake, "Vibrotactile feedback systems: Current trends in rehabilitation, sports and information display," in 2009 IEEE/ASME International Conference on Advanced Intelligent Mechatronics, 2009, pp. 1148-1153. 
[5] Vincent Hayward, Oliver R. Astley, Manuel Cruz-Hernandez, Danny Grant, and Gabriel Robles-De-La-Torre, "Haptic interfaces and devices," Sens. Rev., vol. 24, no. 1, pp. 16-29, Mar. 2004.

[6] "Vibration Motors", Precision Microdrives. [Online]. Available: https://www.precisionmicrodrives.com/vibration-motors.

[7] I. D. Johnston, D. K. McCluskey, C. K. L. Tan, and M. C. Tracey, "Mechanical characterization of bulk Sylgard 184 for microfluidics and microengineering," J. Micromechanics Microengineering, vol. 24, no. 3, p. 035017, 2014.

[8] "Ecoflex ${ }^{\circledast}$ 00-30 Product Information," Smooth-On, Inc. [Online]. Available: https://www.smooth-on.com/products/ecoflex-00-30/.

[9] S. Diridollou et al., "In vivo model of the mechanical properties of the human skin under suction," Skin Res. Technol., vol. 6, no. 4, pp. 214-221.

[10] J. C. Case, E. L. White, and R. K. Kramer, "Soft Material Characterization for Robotic Applications," Soft Robot., vol. 2, no. 2, pp. 80-87, Jun. 2015.

[11] G. Alici, "Softer is Harder: What Differentiates Soft Robotics from Hard Robotics?," MRS Adv., vol. 3, no. 28, pp. 1557-1568, ed 2018.

[12] S. Choi and K. J. Kuchenbecker, "Vibrotactile Display: Perception, Technology, and Applications," Proc. IEEE, vol. 101, no. 9, pp. 2093-2104, Sep. 2013.

[13] S. Bauer, S. Bauer-Gogonea, I. Graz, M. Kaltenbrunner, C. Keplinger, and R. Schwödiauer, "25th Anniversary Article: A Soft Future: From Robots and Sensor Skin to Energy Harvesters," Adv. Mater., vol. 26, no. 1, pp. 149-162, Jan. 2014.

[14] S. Yun, B. B. Kang, and K. Cho, "Exo-Glove PM: An Easily Customizable Modularized Pneumatic Assistive Glove," IEEE Robot. Autom. Lett., vol. 2, no. 3, pp. 1725-1732, Jul. 2017.

[15] E. W. Hawkes, L. H. Blumenschein, J. D. Greer, and A. M. Okamura, "A soft robot that navigates its environment through growth," Sci. Robot., vol. 2, no. 8, p. eaan3028, Jul. 2017.

[16] P. Polygerinos et al., "Soft Robotics: Review of Fluid-Driven Intrinsically Soft Devices; Manufacturing, Sensing, Control, and Applications in Human-Robot Interaction," Adv. Eng. Mater., vol. 19, no. 12, p. 1700016, Dec. 2017.

[17] M. Cianchetti, C. Laschi, A. Menciassi, and P. Dario, "Biomedical applications of soft robotics," Nat. Rev. Mater., vol. 3, no. 6, p. 143, Jun. 2018.

[18] N. Agharese et al., "HapWRAP: Soft Growing Wearable Haptic Device," in 2018 IEEE International Conference on Robotics and Automation (ICRA), 2018, pp. 1-5.

[19] H. Phung et al., "Tactile display with rigid coupling based on soft actuator," Meccanica, vol. 50, no. 11, pp. 2825-2837, Nov. 2015.

[20] A. Marette, A. Poulin, N. Besse, S. Rosset, D. Briand, and H. Shea, "Flexible Zinc-Tin Oxide Thin Film Transistors Operating at $1 \mathrm{kV}$ for Integrated Switching of Dielectric Elastomer Actuators Arrays," Adv. Mater., vol. 29, no. 30, p. 1700880, Aug. 2017.

[21] P. Moseley, J. M. Florez, H. A. Sonar, G. Agarwal, W. Curtin, and J. Paik, "Modeling, Design, and Development of Soft Pneumatic Actuators with Finite Element Method," Adv. Eng. Mater., vol. 18, no. 6, pp. 978-988, Jun. 2016.

[22] G. Agarwal, N. Besuchet, B. Audergon, and J. Paik, "Stretchable Materials for Robust Soft Actuators towards Assistive Wearable Devices," Sci. Rep., vol. 6, Sep. 2016.

[23] R. V. Martinez, A. C. Glavan, C. Keplinger, A. I. Oyetibo, and G. M. Whitesides, "Soft Actuators and Robots that Are Resistant to Mechanical Damage," Adv. Funct. Mater., vol. 24, no. 20, pp. 3003-3010, May 2014. 
[24] B. Mosadegh et al., "Pneumatic Networks for Soft Robotics that Actuate Rapidly," Adv. Funct. Mater., vol. 24, no. 15, pp. 2163-2170, Apr. 2014.

[25] M. A. Robertson and J. Paik, "New soft robots really suck: Vacuum-powered systems empower diverse capabilities," Sci. Robot., vol. 2, no. 9, p. eaan6357, Aug. 2017.

[26] C. Larson et al., "Highly stretchable electroluminescent skin for optical signaling and tactile sensing," Science, vol. 351, no. 6277, pp. 1071-1074, Mar. 2016.

[27] J. Shintake, H. Sonar, E. Piskarev, J. Paik, and D. Floreano, "Soft pneumatic gelatin actuator for edible robotics," in 2017 IEEE/RSJ International Conference on Intelligent Robots and Systems (IROS), 2017, pp. 6221-6226.

[28] D. Rus and M. T. Tolley, "Design, fabrication and control of soft robots," Nature, vol. 521, no. 7553, pp. 467-475, May 2015.

[29] T. Q. Trung and N. Lee, "Flexible and Stretchable Physical Sensor Integrated Platforms for Wearable Human-Activity Monitoringand Personal Healthcare," Adv. Mater., 2016.

[30] A. Hirsch, H. O. Michaud, A. P. Gerratt, S. de Mulatier, and S. P. Lacour, "Intrinsically Stretchable Biphasic (Solid-Liquid) Thin Metal Films," Adv. Mater., vol. 28, no. 22, pp. 4507-4512, Jun. 2016.

[31] S. Nam et al., "A Robust Soft Lens for Tunable Camera Application Using Dielectric Elastomer Actuators," Soft Robot., Aug. 2018.

[32] H. Mößinger, H. Haus, M. Kauer, and H. F. Schlaak, "Tactile feedback to the palm using arbitrarily shaped DEA," presented at the Electroactive Polymer Actuators and Devices (EAPAD) 2014, 2014, vol. 9056, p. 90563C.

[33] R. L. Truby et al., "Soft Somatosensitive Actuators via Embedded 3D Printing," Adv. Mater., 2018.

[34] H. A. Sonar and J. Paik, "Soft Pneumatic Actuator Skin with Piezoelectric Sensors for Vibrotactile Feedback," Front. Robot. Al, vol. 2, 2016.

[35] B. J. P. Mortimer, G. A. Zets, and R. W. Cholewiak, "Vibrotactile transduction and transducers," J. Acoust. Soc. Am., vol. 121, no. 5, pp. 2970-2977, May 2007.

[36] K. A. Kaczmarek, J. G. Webster, P. Bach-y-Rita, and W. J. Tompkins, "Electrotactile and vibrotactile displays for sensory substitution systems," IEEE Trans. Biomed. Eng., vol. 38, no. 1, pp. 1-16, Jan. 1991.

[37] F. Schneider, U. Wallrabe, T. Fellner, and J. Wilde, "Mechanical properties of silicones for MEMS," J. Micromechanics Microengineering Struct. Devices Syst., vol. 18, no. 6, 2008.

[38] J. L. Sparks et al., "Use of silicone materials to simulate tissue biomechanics as related to deep tissue injury," Adv. Skin Wound Care, vol. 28, no. 2, pp. 59-68, Feb. 2015. 


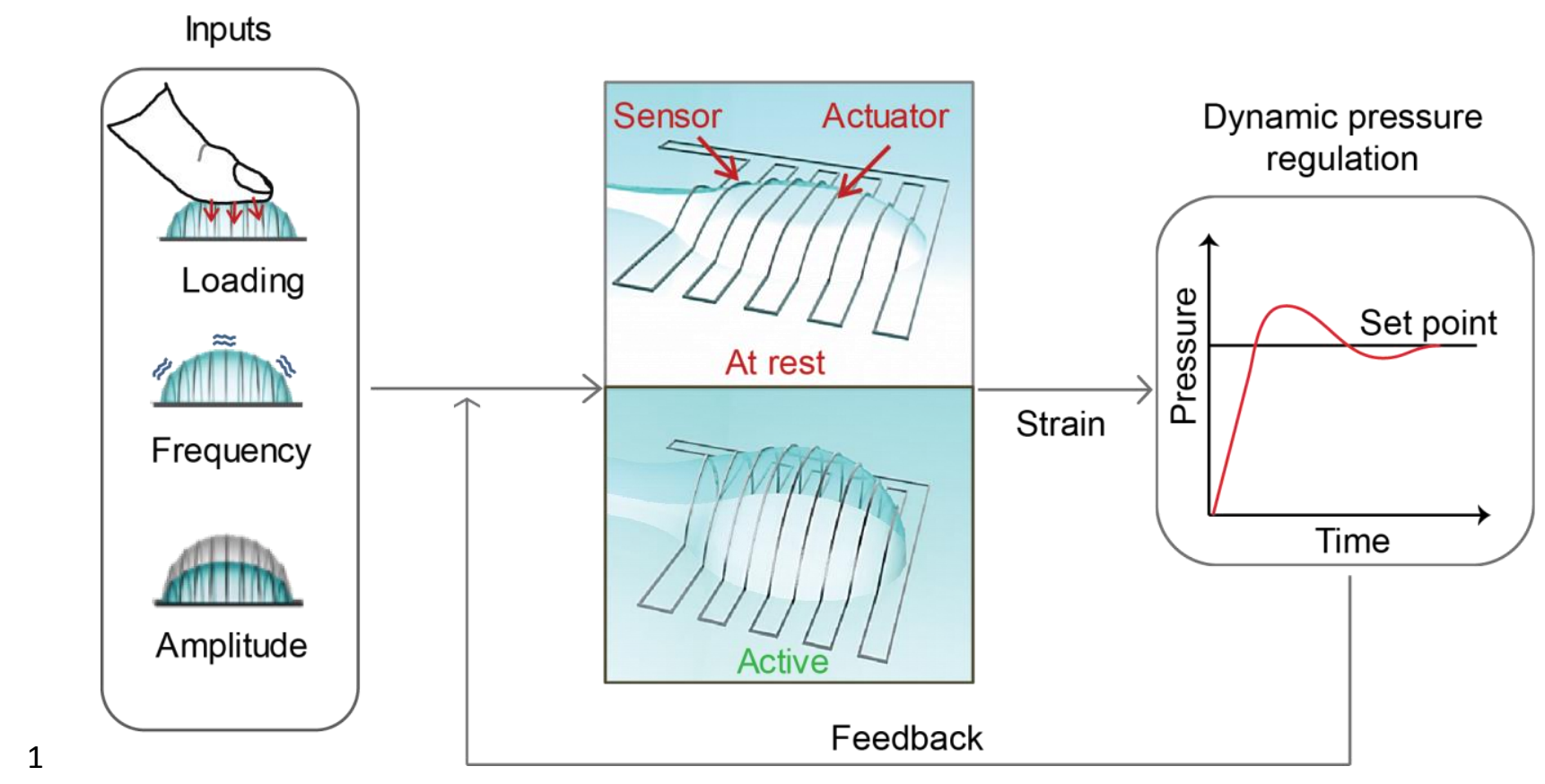

Figure 1. Feedback control loop of soft haptic system. The SPA-skin provides a highly conformal interface. A strain sensor of stretchable metallization can be used as the input to a feedback loop used to control the actuator inflation and exerted force. High-speed data acquisition allows strain sensing and actuation over a range of actuation frequencies from 0 to $100 \mathrm{~Hz}$. 
a

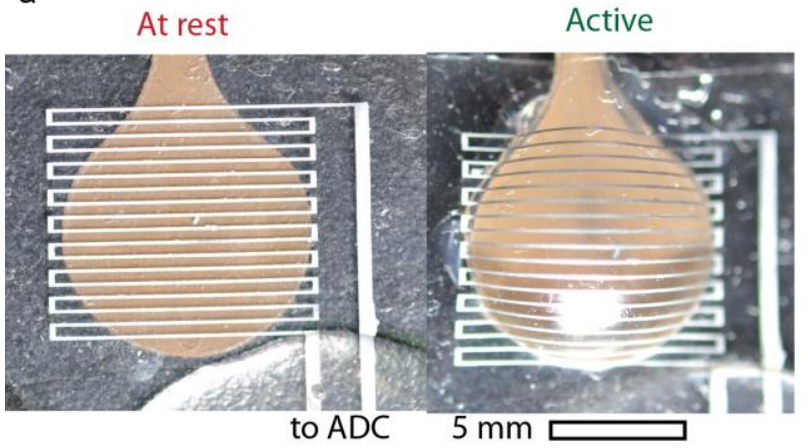

C

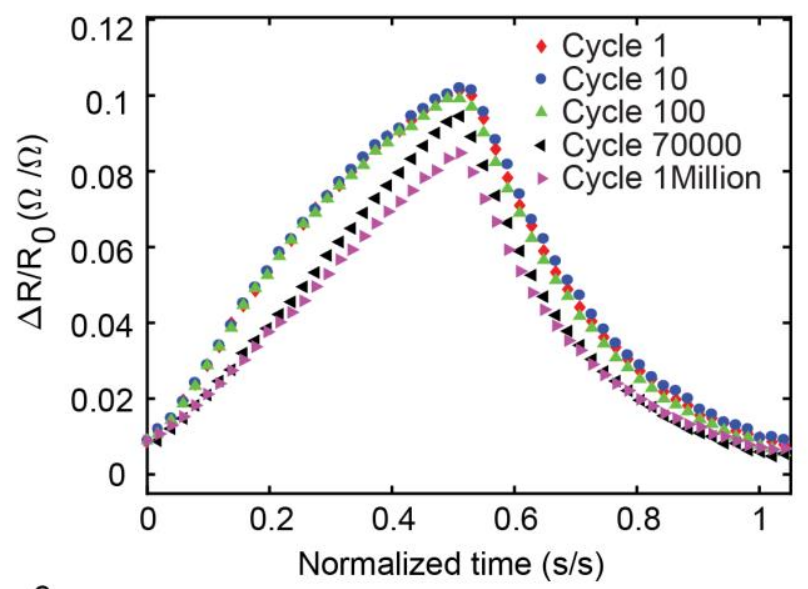

e

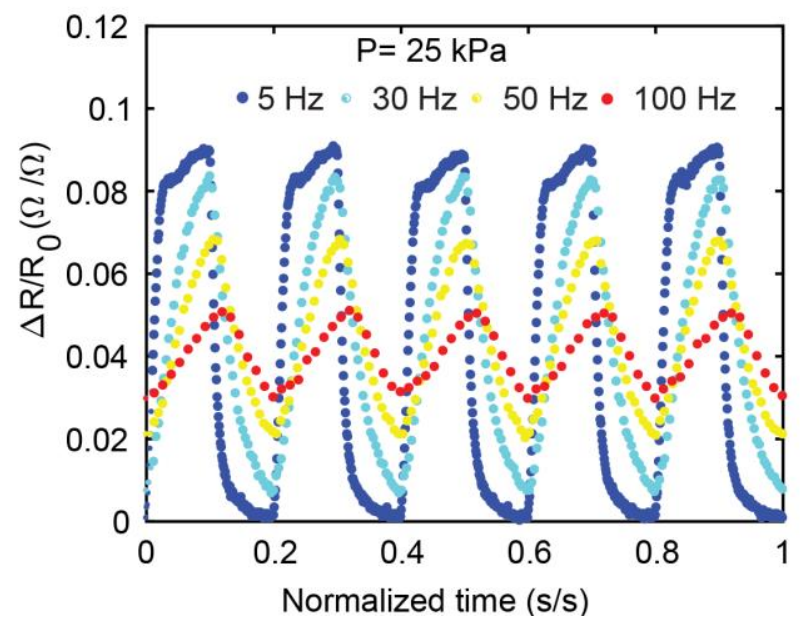

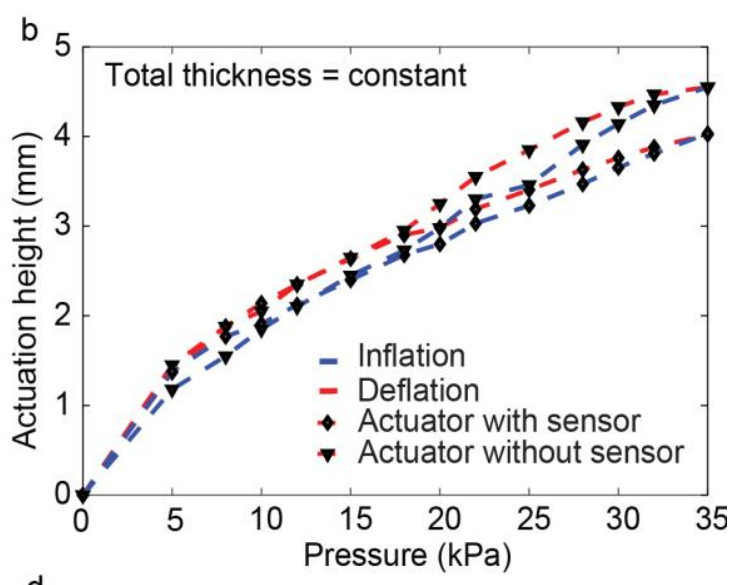

d
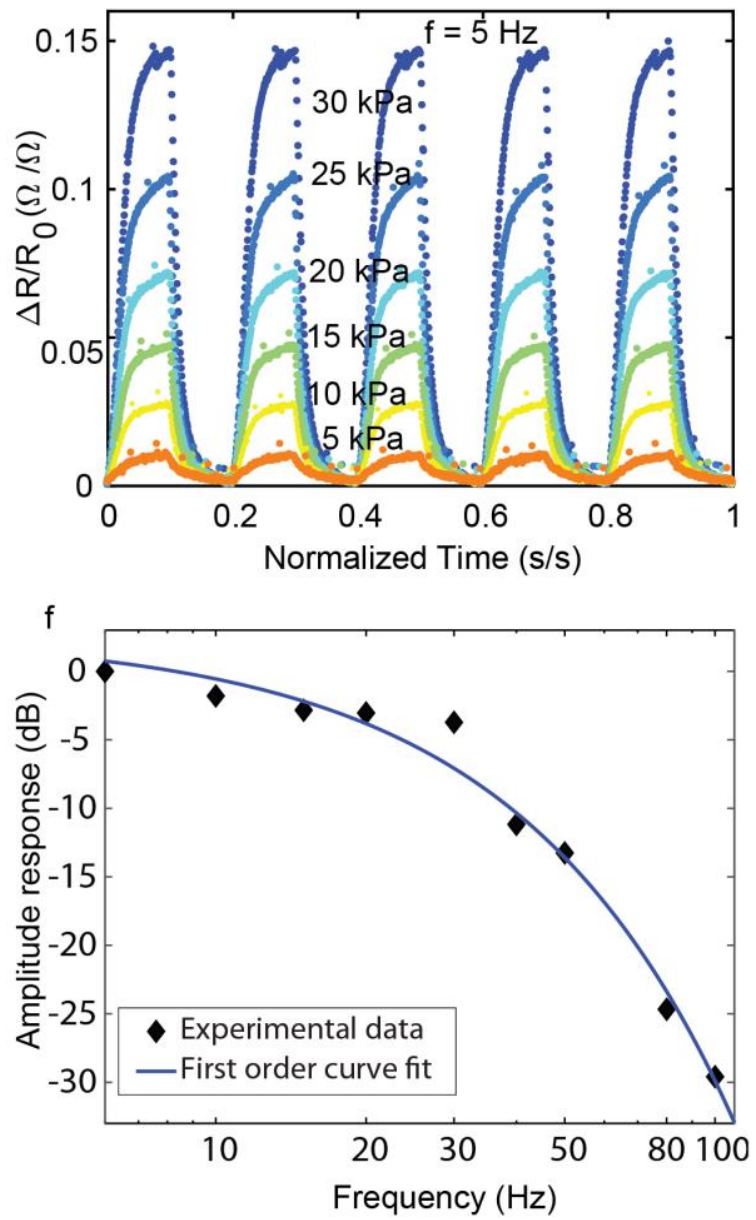

Figure 2. Characterization of SPA-Skin. (a) An SPA-skin prototype before and during inflation. (b) Actuators of the same thickness with and without laminated sensors show little change in performance. (c) Sensor performance over a million cycles. (d) SPA-skin response for variation input pressure set points from $5 \mathrm{kPa}$ to $30 \mathrm{kPa}$ at $5 \mathrm{~Hz}$. (e) SPA-skin response for variation of actuation frequency from $5 \mathrm{~Hz}$ to $100 \mathrm{~Hz}$ at $25 \mathrm{kPa}$ pressure set point. (f) SPA-skin amplitude response for variation in actuation frequency. 
a

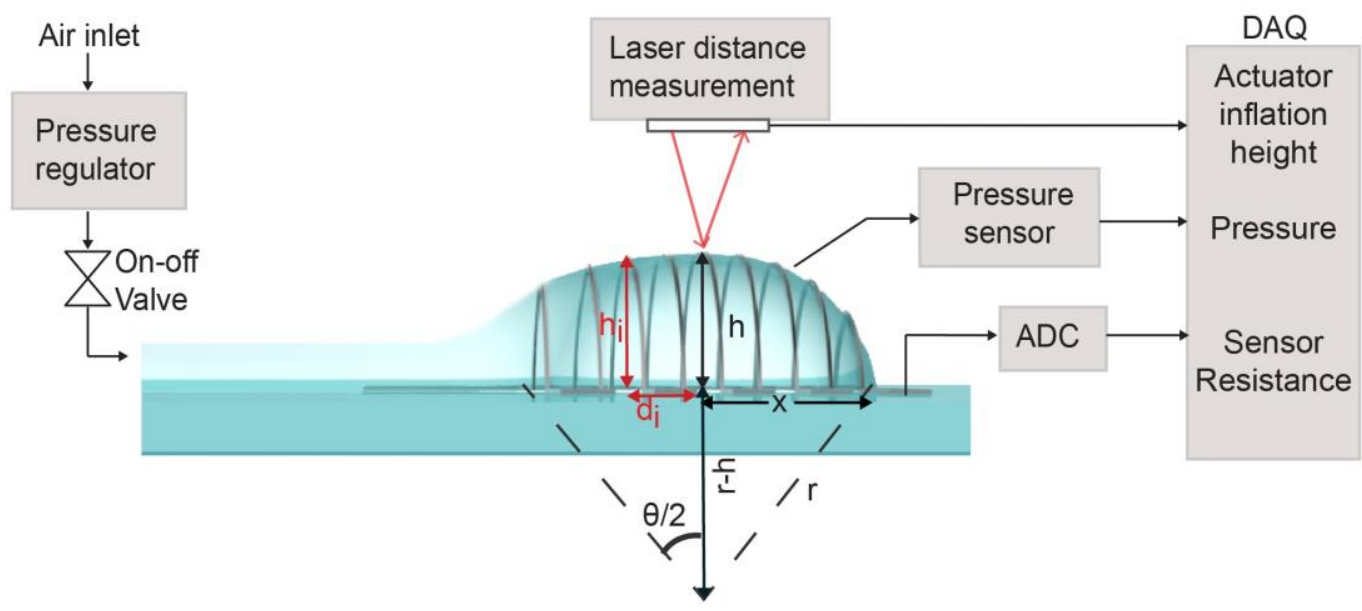

b
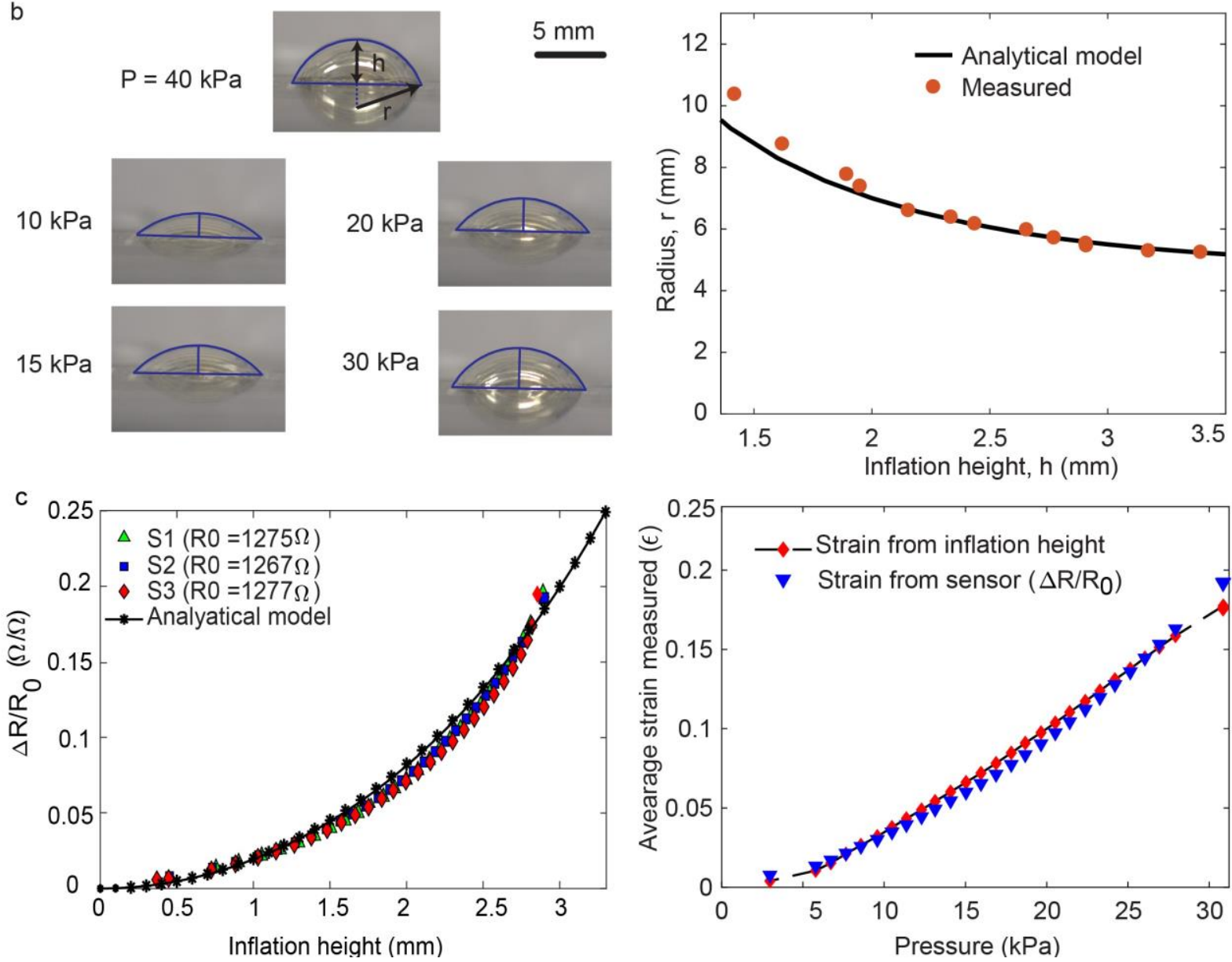

Figure 3. Experimental setup and model. (a) Experimental setup for validating the sensor embedded

SPA model upon actuation, overlaying the geometric parameters used in the analytical model. (b) Images showing the SPA inflation over a range of actuation pressures used to measure the inflation radius (r) and the inflation height $(\mathrm{h})$ for geometrical model validation. The measured inflation radius is compared with the geometric model assuming the SPA inflation geometry as a part of a scooped sphere..

(c) Average measured and computed strains for given inflation heights. (d) Average strain as a function of input pressure (red dots: model, blue dots: experimental strain measured by the resistive sensor). 
a

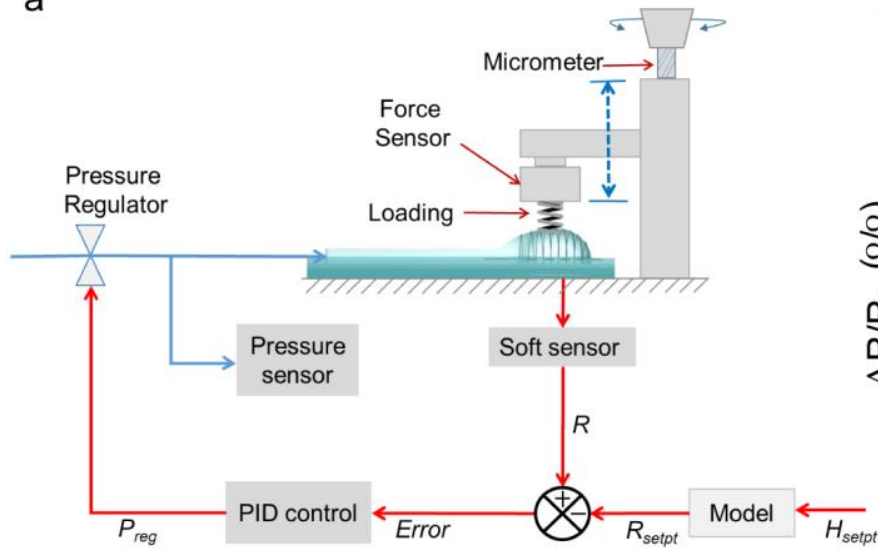

C

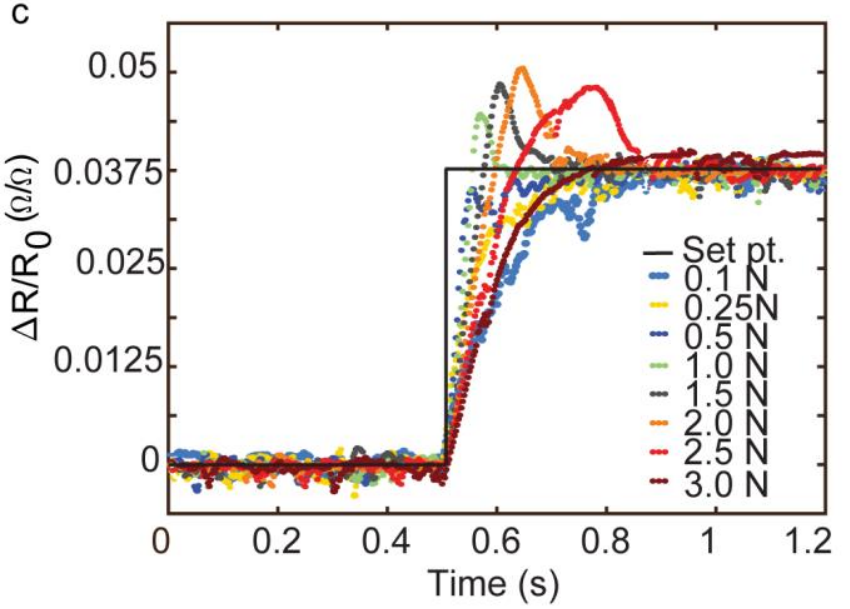

b

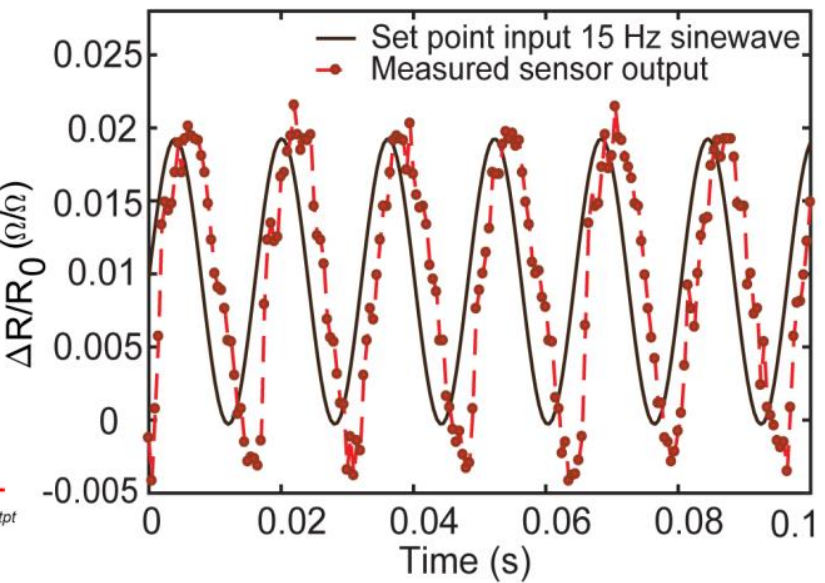

d

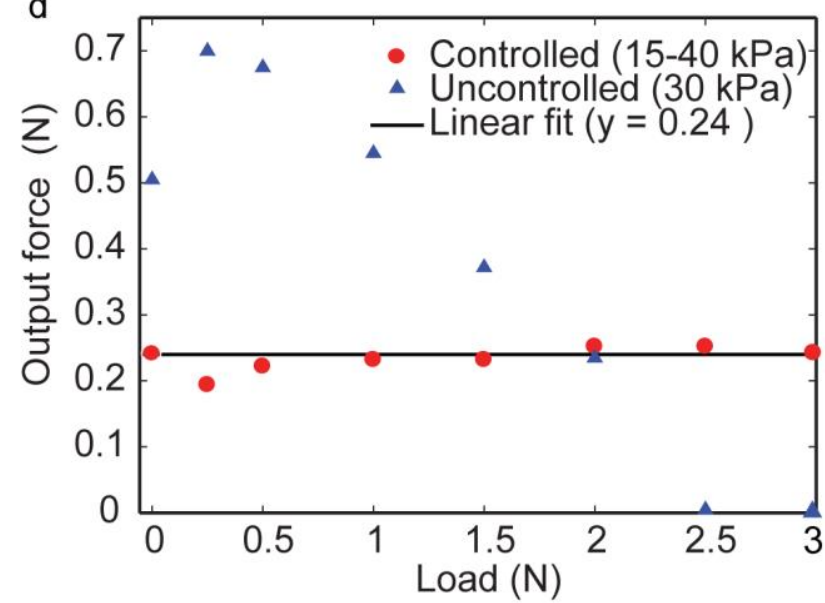

2

3

Figure 4. Testbed for active closed-loop control. (a) Control flow diagram of the SPA-skin with a soft, skin-like material placed between the actuator and an external force sensor, simulating external loading of the sensor against skin. Strain is controlled using PID controller regulate SPA pressure. External pressure sensor limits the maximum input pressure for safe operation. A Vernier scale controls the displacement, and therefore blocked force. (b) Controller tracking a sinusoidal perturbation of set point input at $15 \mathrm{~Hz}$. (c) Controller performance under eight different loading conditions. The controller has a damped response at $3 \mathrm{~N}$ as the pressure required to achieve the set point exceeds the $40 \mathrm{kPa}$ pressure controller safety limit. (d) Effect of loading on the SPA-skin performance when controlled using a pressure sensor and when using an integrated strain sensor to achieve a $0.25 \mathrm{~N}$ blocked force set point. 